changes in the normal lung tissue. It is of great importance to be able to recognize these changes since they may be taken for a recurrence. If this mistake is made and more $\mathrm{X}$-ray treatment given, necrosis and abscess of the lung are likely to follow the second course of irradiation. In a number of patients there are no symptoms or signs, despite marked radiological changes in the lung fields. In others the pulse rate is raised and there is dyspnoea on exertion with paroxysmal attacks of coughing, pain is rare; some begin with signs of an acute pulmonary infection with slight fever and mucopurulent sputum which is diagnosed as influenza or pneumonia. Occasionally there are frequent small haemoptyses or a sero-fibrinous pleurisy with a lymphocytic count in the fluid. The signs and symptoms are usually of short duration but occasionally the syndrome is protracted. The average time of appearance of these radiological changes is four months, but sometimes they appear near the end of treatment or they may be delayed for as long as 18 months after irradiation. Diagnosis is often extremely difficult on account of the variability of the clinical picture and of the physical signs and it depends largely on the interpretation of the X-ray picture.

It is not possible to assess the effect of radiotherapy on survival since the duration of life in untreated cases of carcinoma of the bronchus is so variable. A few patients live for a number of years after radiotherapy but some untreated cases have also been reported to survive for as long as six to seven years after the diagnosis had been made. The average duration of life of patients with an oat-cell or undifferentiated carcinoma is. less than those with a squamous or an adeno carcinoma. In the author's series of cases proved? histologically which had received radical X-raye treatment, the average duration of life of those with an oat-cell or undifferentiated carcinoma wass I 4 months, with a squamous carcinoma 18 month and with an adenocarcinoma 28 months. It hass yet to be seen whether pre-operative irradiation will render more cases operable. The value of palliative X-ray therapy if properly given is un $\vec{\omega}$ doubted. Cough, dyspnoea, haemoptyses and superior vena caval obstruction can often bह alleviated. The general condition improves if these symptoms are relieved, and the patient mayi enjoy some months of normality. A number of patients become well enough to return to work foß a time. The final illness is usually short and deatlos may be fairly sudden from haemorrhage or pneumonia.

\section{BIBLIOGRAPHY}

BROCK (1952), Personal communication.

CLELAND, W. P. (1951) "The Management of the Patient wit Inoperable Cancer of the Chest,' 7. Thor. Surg., 22, No. 5, 5013

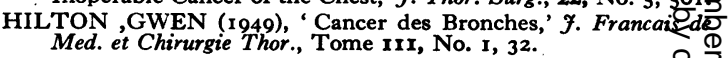

HUGUENIN, R., and FAUVET, J. (1949), 'Cents Canœrs. Bronchiques traités par Radiothérapie,' $¥$. Francais de Medo Chirurgie Thor., Tome IIr, p. 6r.

SMITHERS, D. W. (1953), 'Facts and Fancies about Cances. the Lung,' Brit. med. F., No. 4822 , 1235.

TOD, M. (194I), 'An Inquiry into the extent to which CanceD Patients in Great Britain receive Radiotherapy, John Sherrat and Son, Altrincham.

\title{
SURGERY OF CARCINOMA OF THE BRONCHUS
}

\author{
By L. L. Bromley, M.ChIR., F.R.C.S. \\ Surgeon in Charge of the Thoracic Department, St. Mary's Hospital.
}

Carcinoma of the bronchus is a most lethal form of malignant disease. All too frequently patients present for treatment with the condition hopelessly advanced. It is probably fair to say that not more than one patient out of every four seen by a surgeon is likely to be suitable for a thoracotomy. The results in patients who do have a resection of the lung and its contained tumour are not favourable; the results can be compared with those of resection of the stomach or rectum.
One has not to look far to find the chief reason for the deadliness of carcinoma of the bronchus. Not only is the lung very richly supplied with lymphatics but it is also an extremely vascular w organ; it can be the simplest matter for cancero cells multiplying in the neighbourhood of pulmonary capillaries to enter the circulation, slip away through the heart and settle down at the endes of a systemic arteriole anywhere in the body. 0 Macroscopic growth of tumour in the pulmonaryo 
veins is a well-known finding in the removed specimens and in the post-mortem room; this feature of a lung carcinoma can be compared with a similar finding in many examples of Grawitz tumour of the kidney where growth may extend into the renal vein and even into the vena cava.

Radical surgery can remove the growth in the lung and it can also remove the mediastinal lymph glands. The surgeon can take into account in his operative technique the knowledge that the tumour may spread by the blood stream, but he can, of course, do nothing about spread which has already occurred by this route. Unfortunately, and here is perhaps the most unhelpful feature of this tumour, the likelihood of blood-borne metastases bears no true relationship to the size or age of the tumour. Thus a minute primary may produce widespread secondaries; this, however, is the exception rather than the rule and the dictum that successful surgery must be early surgery still holds true.

\section{Selection of Patients for Surgery}

As in the present state of our knowledge the patient's chief, if not only, hope of survival is by removal of the lung, it will be reasonable to discuss the contraindications to operation rather than the indications. Some contraindications are absolute, some are relative and these will be considered shortly. There remains with every patient the assessment of that patient's own personal fitness to withstand a pneumonectomy and this judgment may be difficult to give. Although a lobectomy may be the operation of choice, one should always make the assessment in terms of a pneumonectomy as when the chest is opened circumstances may demand the latter procedure. Generally speaking, it is fairly easy to make a decision, but in the more elderly emphysematous patient difficulties arise. Unfortunately lung function studies are only within the reach of a few and in the end it is often the surgeon's personal judgment that makes the final decision.

\section{Pre-operative Investigations}

Besides the history and physical examination and standard X-rays of the chest, there are other important investigations to be carried out. On the results of these not only will the clinical diagnosis be verified but an overall assessment of operability made.

\section{(i) Barium Swallow}

Both right and left main bronchi and lower lobe bronchi are in close relation to the oesophagus and glandular enlargement around these bronchi and in the mediastinum may be demonstrated by barium swallow. This investigation is well worth making a routine as glands may sometimes be demonstrated by a swallow when evidence of their presence is lacking on bronchoscopy.

\section{(ii) Screening}

This should always be performed in order to check upon movements of the diaphragm.

\section{(iii) Bronchoscopy}

This is the most important single investigation and should never be omitted. Even if all the $\vec{\circ}$ other features of the clinical picture suggest that $\overrightarrow{ }$ this will be a superflous investigation, it cannot ${ }_{\sigma}^{\omega}$ be too strongly stressed that it is essential to per-form it. Anaesthesia: Either local or general 3 may be used. Local is given by a premedication of Omnopon gr. $1 / 3$ and Scopolamine gr. $1 / 150.0$ This is followed by an Amethocaine tablet sucked $\omega_{\infty}$ half an hour before the examination; 3 cc. of $\%$ I per cent. Amethocaine are dripped slowly over $\vec{\sigma}$ the back of the tongue and I cc. of the sameo solution is injected into the trachea through the front of the neck. To supplement the basal nar- $\square$ cosis in a still wideawake patient $5^{\circ} \mathrm{mgm}$. of ${ }_{\%}^{\Phi}$ Pethidine may be injected intravenously ten $\frac{\Phi}{3}$ minutes before the examination. For a genergl anaesthetic the same premedication is given argd then Pentothal is given intravenously in conjun $-\overrightarrow{0}$ tion with a quick-acting, short-lasting relaxagt such as Scoline. Oxygen is given either down the side tube of the bronchoscope or through a tightfitting tube with ' bag' inflation at intervals during the examination. One cannot lay down hard and fast rules as to which method of anaes- $\mathbb{D}$ thesia is preferable. It is recommended that both techniques should be learned and then the method suited to the individual patient. More relaxation $\frac{3}{5}$ is obtained with general anaesthesia and often a better view is obtained of that difficult region to see, the upper division of the left upper lobe $\frac{3}{3}$ bronchus.

Before passing the instrument into the trachea 3 note should be taken of movements of the vocalo cords, the patient being under local anaesthesia. $\overline{3}$ A left recurrent laryngeal palsy is a common $\mathrm{O}$ sequela of a carcinoma of the left lung. Where doubt exists as to the presence or absence of $a \frac{}{0}$ recurrent palsy it is recommended that indirect laryngoscopy be performed on another occasion $N$ with no local anaesthetic used. Sluggish move- N ments of the cords are often seen with local N anaesthesia and can be wrongly interpreted.

The trachea and carina are inspected in turn. Both these may be deformed by pressure due to 0 enlarged secondary glands. Enlarged inflammatory glands do not distort the air passages in adults, thus it is a fair supposition that if such 
distortion is present then it is due always to secondary glands. There are many anatomical variants in the shape of the carina and it is a matter of experience to judge when actual deformity is present. The main point, however, to note is that the widening that occurs with enlarged glands is subcarinal, and this subcarinal widening is reflected in the shape of one or other or both of the main bronchi, producing some narrowing. The surgeon should next examine both bronchial trees in turn, preferably the normal side first and then the suspected side; the use of a right-angled telescope aids fuller inspection of the upper lobe orifices.

There may be direct evidence of a carcinoma; this can present as a pale grey, pink or white friable warty excrescence partially filling the lumen of a bronchus or completely obliterating it. This mass will bleed freely when touched. Or the presentation may be that of a rapid narrowing of the bronchus with a granular or nodular appearance of the mucosa.

Indirect evidence of a carcinoma will be revealed by a deformity of the bronchus by exobronchial compression from glands, either as an inward bulging of one wall or a circumferential stenosis, the mucosa at the level of this stenosis appearing macroscopically normal. Deformity of secondary carinae is again indirect evidence of intrapulmonary glandular enlargement and thence of carcinoma.

A biopsy should be taken in all cases where tumour is visible. And in some cases where only indirect evidence of a tumour is seen it may be possible to obtain positive histology from a biopsy taken from the normal looking mucosa in the region of the deformity; submucous spread of cancer cells may have reached this level. A note of warning must be sounded, however, 'blind' biopsies, e.g. from the depths of a lower lobe bronchus, or deep biopsies of normal looking bronchi should be avoided or otherwise very carefully done. It is not difficult to injure a bronchial artery or a pulmonary artery and produce most disturbing haemorrhage.

A note should be made of the amount and quality of secretions in the bronchial tree, both in relation to the region of the growth and also in the 'sound' lung. These secretions give an indication of the condition of the bronchial mucous membrane beyond the limits of vision. In those patients with emphysema and bronchitis evidence of purulent secretion on the 'sound' side may help in making the decision for or against a thoractomy.

Collection of sputum. At bronchoscopy a specimen of sputum should be collected, preferably from the region under suspicion, and this specimen should be examined for malignant cells. In suspected peripheral carcinomas where there is frequently no bronchoscopic evidence of tumour at all and also very little secretion, it is worthwhile to inject $5 \mathrm{cc}$. of normal saline into the suspected lobe, using the sucker for the injection and then turning back to suction as the patient coughs, a good specimen can thus be obtained from the part that matters (see paper on cytological techniques by J. L. Pinniger).

Summing up then, the value of bronchoscopy, it can strengthen or confirm a clinical diagnosis and can give evidence of inoperability as well as of operability.

\section{(iv) Bronchography}

Only very rarely is bronchography of help in diagnosis. In the main bronchus type of tumour it is probably never necessary as bronchoscopy will give more information. Where it may occasionally be of value is the differentiating between lesions more at the periphery of the lung where the diagnosis rests between a peripheral carcinoma, not well circumscribed, and a slowly resolving area of pneumonitis. Evidence of bronchial obstruction of an air passage of a small order will be in favour of a carcinoma. One may also sometimes regret having done a bronchoo graphy as the lipiodol residue may obscure the detail of the lesion under observation.

\section{(v) Tomography}

Tomography is occasionally of value, particularly in the elucidation of rounded opacities and in giving information of bone involvement better than a penetrating film.

Two further investigation procedures should be mentioned. Not infrequently patients present with conclusive evidence of a bronchial carcinoma, apparently operable and confined to the chest, yet their poor general condition suggests the presence of secondaries. Metastases notoriously lurk in the suprarenals and to a lesser extent in the liver. A pneumogram to outline the suprarenals is not a difficult technical procedure to carry out, using the precoccygeal route for the injection of the air, and can be valuable in this type of case. Also valuable and seriously to be considered on occasions is an E.U.A. of the abdomen and pelvis, and if still in doubt to proceed to a small laparotomy in order to examine properly a suspect liver.

\section{Contraindications to Thoracotomy}

(i) Distant extrathoracic metastases clearly contraindicate surgery. It is not believed that there is a place for palliative pneumonectomy as there is a place for palliative resection of the stomach or rectum. The only exceptional circumstance where 
this might be considered is when the patient is bleeding grossly from the primary tumour, having in fact large and terrifying haemoptyses, and whose s co idary deposits are small and symptomless. This circumstance is of great rarity. Palliative surgery should not be considered where there is gross sepsis, for example, following the central breakdown of a large peripheral tumour. This seps s can be satisfactorily controlled by postural drainage and by antibiotics.

(ii) Left recurrent laryngeal nerve palsy is another absolute contraindication to thoracotomy as this denotes either direct infiltration of the nerve as it courses below the aortic arch or secondary pressure on it from a mass of involved glands in a similar situation.

(iii) Diaphragmatic paralysis. Where the primary tumour arises in the upper lobe of the lung the phrenic nerve will be involved in the region of the great vessels at the base of the heart and thoracotomy is contraindicated. Where, however, the phrenic nerve is involved by infiltration or pressure from a lower lobe growth a pneumonectomy may still be a possibility, for, if other things are satisfactory, it is possible to resect a wide area of pericardium on which the phrenic nerve lies.

(iv) Chest wall involvement. There are, of course, degrees of this. It occurs with peripheral growths and there may be erosion of only one or two ribs as seen radiologically. In actual practice the extent of bone involvement is always very much greater than is demonstrated by $\mathrm{X}$-rays. If the primary tumour is still small then a pneumonectomy with removal of the chest wall locally is a justifiable operation. With any more extensive involvement surgery is contraindicated, although extreme pain may influence the surgeon in his decision. He must, however, realize that a radical operation is an improbability, as once the chest wall becomes involved in growth the lymphatics leading to the axilla are open to transmit cancer cells and a total axillary glandular clearance becomes a theoretical necessity but an almost certain practical impossibility.

(v) Evidence of enlarged mediastinal glands. As already stated, evidence of these is found on bronchoscopy and on barium swallow, and where such enlargement exists it can be assumed to be due to malignant involvement. Furthermore, it is found that the degree of involvement is usually much more than is expected. Enlarged paratracheal glands, distortion of the carina and of the oesophagus all contraindicate thoracotomy.

(vi) Pleural effusion. A blood-stained effusion certainly contraindicates exploration, whilst a straw-coloured effusion generally does so, unless there is strong evidence to suppose that the effusion is an inflammatory one and secondary to infection in the lung distal to the obstruction due to the carcinoma.

(vii) Bronchoscopic evidence of tracheal spread. With a growth arising in one of the main bronchi $\overrightarrow{\vec{F}}$ upward extension on to the carina or into the $\frac{\overrightarrow{0}}{0}$ lower part of the trachea will almost certainly $\frac{}{0}$ contraindicate operation. Very rarely, with small $\overline{\bar{c}}$. tumours, carinal resection and reconstruction of $\vec{\nabla}$ the lower end of the trachea and the remaining main bronchus is a possibility.

(viii) Pancoast tumours. This type of peripheral $\overrightarrow{0}$ carcinoma which grows at the apex of the lung $\vec{\overrightarrow{ }}$ and involves the cervical sympathetic chain and the $\tilde{\sigma}^{\circ}$ first dorsal nerve is not surgically removable, $\frac{D}{0}$ usually because of involvement of the sides of the $\frac{0}{3}$ bodies of the upper thoracic vertebrae.

\section{Operative Technique}

There is not space to discuss anaesthesia in any detail; the essential features are:

(I) Controlled respiration through an endotracheal cuff tube.

(2) Light anaesthesia so that the patient is $\vec{\nabla}$ quickly ' round' after the operation and $\mathbb{\varnothing}$ able to cough.

(3) Adequate oxygenation at all times.

(4) Control of secretions at all times. Thoracotomy may be performed in the lateral of in the face-down position; in the former some sort of bronchial blocker is a great advantage blocking off the affected lung and preventing spread of infected secretions whilst the lung is being handled. The chest is opened through the bed of the 6th rib and an assessment is made $\frac{D}{\perp}$

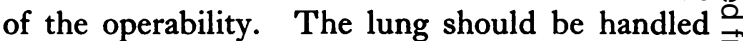
as little as possible, being gently mobilized or $\overline{ }$ retracted in order to palpate the hilum or get a direct view of it. The bronchus, pulmonary artery and the veins are examined, it being ascertained that each can be divided proximal to the $\frac{0}{3}$ tumour. The degree of glandular enlargement in the mediastinum is difficult to assess fully at $\frac{3}{3}$ this stage. Probably the most important step comes next once the condition has been deemed $\frac{9}{3}$ operable, and this is the placing and tying of a ligature around the pulmonary vein, superior or inferior, corresponding to the lobe in which the $\frac{D}{0}$ tumour arises. Early ligature of this vein is strongly recommended so that there is then less $N$ likelihood of squirting off malignant emboli to- N wards the heart during subsequent manipulation $N$ of the lung (Aylwin, 195I).

The order in which the rest of the hilar structures are taken does not greatly matter; if there $\stackrel{\varrho}{\subset}$ is any question of failure to control spill of secre- $\mathbb{D}$ tion the bronchus should be clamped at an early? stage. It should subsequently be sutured close 
to the carina and stainless steel wire is recommended as suture material (Belsey, 1946). After the lung has been removed from the chest a clearance is made of all visible mediastinal glands, including, of course, the subcarinal group, and also those of the paratracheal group. These are much easier to dissect out on the right side of the chest; on the left side the aortic arch impedes somewhat the dissection. If possible the left recurrent laryngeal nerve should be preserved, but if its preservation means inadequate gland removal then it should be sacrificed. It is often a pleasant surprise to find that great numbers of enlarged mediastinal glands removed are found on section to be only inflammatory. This fact is heartening to the surgeon and pneumonectomy should never be abandoned when glands are found at the original assessment and these glands are not obviously malignant.

With growths very adjacent to the hilum a formal wide opening of the pericardium anteriorly with intra-pericardial ligation of the vessels is advised (Allison, I946). Many more cases become operable if this technique is practised. It entails resection of the pericardium around the hilum and allows ligature of the veins flush with the left auricle. There is little, if any, more immediate risk entailed if this technique is used for the main bronchus types of tumour, whilst the post-operative course is also not made more difficult except that cardiac irregularities are more prone to develop, but these may be easily controlled with digitalis. It may well become a routine procedure.

\section{Lobectomy or Pneumonectomy}

In the present state of our knowledge it would appear that lobectomy is a satisfactory procedure for a peripheral carcinoma, if this is small or even if it is large, where no obvious secondary glands are present. Results do appear to justify this less radical operation, particularly in older people where much needed lung tissue can be preserved. Peripheral growths tend to metastasize a little more by the blood stream rather than by lymphatics giving further weight to the argument for lobectomy. It must be stated, however, that if any evidence, direct or indirect, of a carcinoma is seen on bronchoscopy, then it is unlikely that lobectomy will be technically possible.

It is appropriate to mention here the surgeon's attitude to the symptomless round shadow in the lung. In the absence of strong circumstantial evidence that this is a tuberculoma, carcinoma should be the first diagnosis and in any adult patient thoracotomy should be advised with a lobectomy the aim.
Results

\section{Operability}

Two figures must be considered here. Firstly,,$\stackrel{\varrho}{c}$ of those patients submitted to a thoracotomy 60 per cent. to 70 per cent. are found to be operable and a resection is performed. This figure is on the increase, undoubtedly because the art of selecting patients or surgery has improved, but the technique of intrapericardial dissection has $\mathbb{Q}$ also played a part.

The second figure, how many of the total number of patients suffering from a bronchial carcinoma are suitable for a thoracotomy, is very $\vec{\omega}$ difficult to obtain. It is probably in the neigh-o bourhood of 15 to 20 per cent. The figureso given by surgeons and by physicians show great? variation. With earlier diagnosis, particularly․ㅡ with the use of mass miniature radiography, thisic figure may be expected to improve (Brock, $1950 ; 0$ Churchill, 1950).

\section{Operative Mortality}

This is ro to I 5 per cent., being very much less than it was ten years ago, due to improvedo anaesthesia and better preparation of the patient. $\Phi$

\section{Follow-up After Resection}

Most recurrences occur in the first 12 montasso after operation, a 50 per cent. mortality may be expected in this year. For those surviving a year the outlook becomes very much more hopeful, and the most recent figures available show a 30 per cent. five-year survival. This figure compareso quite favourably with carcinoma of the stomach.

Where improvement is sadly needed is bringing $\rightarrow$ more patients earlier to the surgeon, that is, theo을 thoractomy rate should be increased from its present level of 15 to 20 per cent., and it is only? by earlier diagnosis that this will be achieved.

\section{Surgery for the Inoperable Patient}

The only indication for palliative surgery is the $\frac{5}{3}$ relief of intractable pain due to involvement of the chest wall. Of value is injection of the inter- 3 . costal nerves posteriorly with a long-acting local anaesthetic, Efocaine or Proctocaine, though there is a danger of some of this solution entering the theca and damaging the cord. Formal division of the intercostal nerves may be an effective $N$ procedure where the chest wall is involved ${ }_{N}$ laterally or anteriorly. A very high cervical cor- 옹 dotomy may be performed, but owing to the $\omega$ arrangement of the fibres forming the spino-? thalamic tract, which cross over many segments higher than their point of entry into the cord, it $\Phi$ is not usually possible to achieve complete relief from pain unless the highest intercostal involved $\frac{0}{0}$ 
is about $\mathrm{T}_{4}$. It is of no value in Pancoast tumours. Leucotomy must be considered for some patients; this can save sometimes quite untellable misery. In most cases, however, pain due to chest wall invasion can be quite satisfactorily controlled by morphia.

\section{BIBLIOGRAPHY}

ALI.ISON, P. R. (19+6), F. Thoracic Surgery, 15, 99.

AYLWIN, J. A. (I95I), Thorax, 6, 250.

BELSEY, R. (1946), Thorax, I, 39.

BROCK, R. (. (1950), Brit. Med. Ұ., i, i I6.

CHURCHHLL, E. D., SWEE'T, R. H., SOLT'TER, L., and SCANNELL, J. (j. (1950), \%. Thoracic Surgery, 20, 349.

\title{
CLINICAL SECTION
}

\section{POLYCYSTIC DISEASE OF THE KIDNEYS}

\author{
By F. M. Hull \\ St. Mary's Hospital London
}

Polycystic disease of the kidneys was first described in 1700 by Littré of Paris. Its familial nature was suggested in 1843 by Adamkiewicz, and is now generally accepted. 'The kidneys, in polycystic disease, become converted into a mass of cysts. In some cases this may be a cause of stillbirth or death in infancy, but in others the condition remains latent until early middle age. Rarely does the disease declare itself between infancy and middle age and in some cases the renal lesions may be insufficient to impair renal function, in which case the condition may only be found at autopsy.

The incidence of the disease can be seen from the accompanying figures

Cases admitted to hospital: I in 3,500 .

Cases found at autopsy: $I$ in 375 .

The two striking points shown by these figures are the difference between the number of cases found clinically and those found at autopsy, and the two distinct age groups in which the disease occurs.

Polycystic kidney disease may be divided into two types : the infantile type and the adult type. The latter may be sub-divided according to whether the condition produces symptoms or not, into clinical and subclinical groups.

In the infantile type of polycystic kidney the child is either stillborn or dies in infancy; he rarely lives longer than a year. In some cases the kidneys may be so large as to hinder labour and occasionally delivery is only possible after evisceration of the foetus. The kidneys are grossly enlarged and on section show a honeycomb

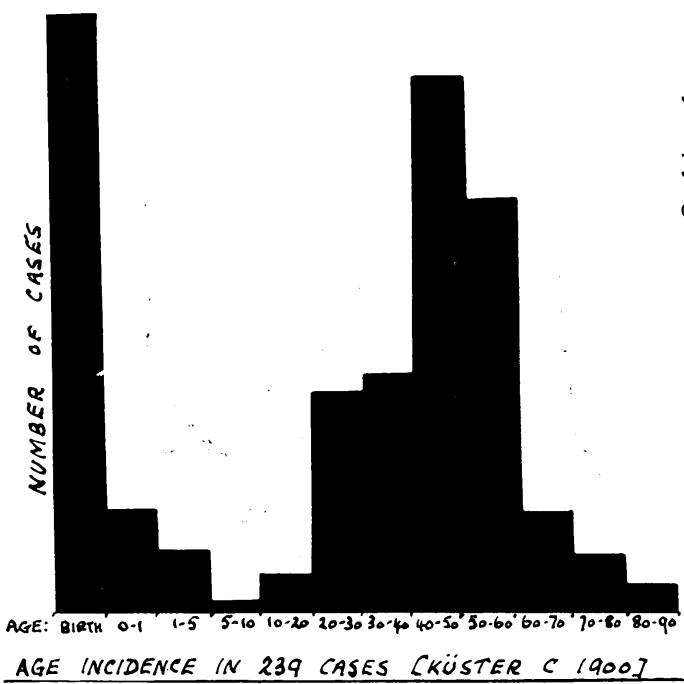

FIG. I.-Diagram showing the age incidence of polycystic disease of the kidneys in 239 cases (after Küster).

appearance with small cysts of fairly regular size distributed throughout the cortex and medulla, the number of nephrons being markedly reduced. Too little renal parenchymal tissue exists to permit more than a few months of life.

In the clinical adult type ot polycystic kidney, the kidneys are greatly enlarged. (One pair of kidneys on record weighed over $7 \mathrm{~kg}$. each.) $\stackrel{\oplus}{?}$ The surface of the kidney is nodular and the cut 